PROCEEDINGS OF THE

AMERICAN MATHEMATICAL SOCIETY

Volume 133, Number 5, Pages 1299-1305

S 0002-9939(04)07664-6

Article electronically published on November 19, 2004

\title{
SYMMETRIZED CHEBYSHEV POLYNOMIALS
}

\author{
IGOR RIVIN
}

(Communicated by Juha M. Heinonen)

\begin{abstract}
We define a class of multivariate Laurent polynomials closely related to Chebyshev polynomials and prove the simple but somewhat surprising (in view of the fact that the signs of the coefficients of the Chebyshev polynomials themselves alternate) result that their coefficients are non-negative. As a corollary we find that $T_{n}(c \cos \theta)$ and $U_{n}(c \cos \theta)$ are positive definite functions. We further show that a Central Limit Theorem holds for the coefficients of our polynomials.
\end{abstract}

\section{INTRODUCTION}

Let $T_{n}(x)$ and $U_{m}(x)$ be the Chebyshev polynomials of the first and second kinds, respectively. We define the following Laurent polynomials (which, for lack of a better name, we call the symmetrized Chebyshev polynomials):

$$
\begin{aligned}
& R_{n}\left(c ; x_{1}, \ldots, x_{n}\right)=T_{n}\left(\frac{c \sum_{i=1}^{k}\left[x_{i}+\frac{1}{x_{i}}\right]}{2 k}\right), \\
& S_{n}\left(c ; x_{1}, \ldots, x_{n}\right)=U_{n}\left(\frac{c \sum_{i=1}^{k}\left[x_{i}+\frac{1}{x_{i}}\right]}{2 k}\right) .
\end{aligned}
$$

The function $R_{n}$ arises in the enumeration of conjugacy classes in the free group on $k$ generators; more specifically, there is the following result:

Theorem 1 ([Rivin99]). The number of cyclically reduced words of length $k$ in $F_{r}$ homologous to $e_{1}\left[a_{1}\right]+\cdots+e_{r}\left[a_{r}\right]$ is equal to the coefficient of $x_{1}^{e_{1}} \cdots x_{r}^{e_{r}}$ in

$$
2(\sqrt{2 r-1})^{k} R_{k}\left(\frac{r}{\sqrt{2 r-1}} ; x_{1}, \ldots, x_{r}\right)+(r-1)\left[1+(-1)^{k}\right] .
$$

Received by the editors February 7, 2003 and, in revised form, January 9, 2004.

2000 Mathematics Subject Classification. Primary 05C25, 05C20, 05C38, 41A10, 60F05.

Key words and phrases. Chebyshev polynomials, positivity, central limit theorem.

These results first appeared in the author's 1998 preprint "Growth in free groups (and other stories)", but seems to be of independent interest. The positivity result was preprint math.CA/0301210, but there appears to be no reason to separate it from the limiting distribution result, and many reasons to keep them together. The author would like to thank the Princeton University Mathematics Department for its hospitality, and the NSF DMS for its support. He would also like to thank the anonymous referee for useful comments on an earlier version of this paper. 
Remark 2. The rescaled Chebyshev polynomial $T_{k}(a x) / a^{k}$ is called the $k$-th Dickson polynomial $T_{k}(x, a)$ (see [Schur73]).

The coefficients of generating functions of combinatorial objects are non-negative. So one is led to wonder for which values of $c$ are the coefficients of $R_{n}\left(c ; x_{1}, \ldots, x_{k}\right)$ and $S_{n}\left(c ; x_{1}, \ldots, x_{k}\right)$ non-negative. In this note we give an essentially complete answer when $k=1$ (Theorem [3) and a partial answer when $k>1$ (Theorem 7 and remark following that theorem). We note that this implies the positive definiteness of the functions $T_{n}(c \cos \theta)$ and $U_{n}(c \cos \theta)$ (Corollary 6). We also write down an explicit formula (Eq. (16)) for the coefficients of $R_{n}$ and $S_{n}$. Furthermore, we use the positivity and tools of probability theory to analyze the distribution of the coefficients of $R_{n}$ to prove a central limit theorem (Theorem 8).

\section{Some facts about Chebyshev polynomials}

The literature on Chebyshev polynomials is enormous; [Rivlin90] is a good place to start. Here, we shall supply the barest essentials in an effort to keep this paper self-contained.

There are a number of ways to define Chebyshev polynomials (almost as many as there are of spelling their inventor's name). A standard definition of the Chebyshev polynomial of the first kind $T_{n}(x)$ is

$$
T_{n}(x)=\cos n \arccos x .
$$

In particular, $T_{0}(x)=1, T_{1}(x)=x$. Using the identity

$$
\cos (x+y)+\cos (x-y)=2 \cos x \cos y
$$

we immediately find the three-term recurrence for Chebyshev polynomials:

$$
T_{n+1}(x)=2 x T_{n}(x)-T_{n-1}(x) .
$$

The definition of Eq. (4) can be used to give a "closed form" used in Section 3

$$
T_{n}(x)=\frac{1}{2}\left[\left(x-\sqrt{x^{2}-1}\right)^{n}+\left(x+\sqrt{x^{2}-1}\right)^{n}\right] .
$$

Indeed, let $x=\cos \theta$. Then

$$
\left(x-\sqrt{x^{2}-1}\right)^{n}=\exp (-i n \theta)
$$

while

$$
\left(x+\sqrt{x^{2}-1}\right)^{n}=\exp (i n \theta)
$$

so that

$$
\frac{1}{2}\left[\left(x-\sqrt{x^{2}-1}\right)^{n}+\left(x+\sqrt{x^{2}-1}\right)^{n}\right]=\Re \exp (\operatorname{in} \theta)=\cos n \theta .
$$

We also define Chebyshev polynomials of the second kind $U_{n}(x)$, which can again be defined in a number of ways, one of which is

$$
U_{n}(x)=\frac{1}{n+1} T_{n+1}^{\prime}(x) .
$$

A simple manipulation shows that if we set $x=\cos \theta$, as before, then

$$
U_{n}(x)=\frac{\sin (n+1) \theta}{\sin \theta} .
$$


In some ways, Schur's notation $\mathcal{U}_{n}=U_{n-1}$ is preferable. In any case, we have $U_{0}(x)=1, U_{1}(x)=2 x$, and otherwise the $U_{n}$ satisfy the same recurrence as the $T_{n}$, to wit,

$$
U_{n+1}(x)=2 x U_{n}(x)-U_{n-1}(x) .
$$

From the recurrences, it is clear that for $f=T, U, f_{n}(-x)=(-1)^{n} f(x)$, or, in other words, every second coefficient of $T_{n}(x)$ and $U_{n}(x)$ vanishes. The remaining coefficients alternate in sign; here is the explicit formula for the coefficient $c_{n-2 m}^{(n)}$ of $x^{n-2 m}$ of $T_{n}(x)$ :

$$
c_{n-2 m}^{(n)}=(-1)^{m} \frac{n}{n-m}\left(\begin{array}{c}
n-m \\
m
\end{array}\right) 2^{n-2 m-1}, \quad m=0,1, \ldots,\left[\frac{n}{2}\right] .
$$

This can be proved easily using Eq. ([6]).

\section{Analysis of the functions $R_{n}$ And $S_{n}$}

In view of the alternation of the coefficients, the appearance of the Chebyshev polynomials as generating functions in Theorem 1 seems a bit surprising, since combinatorial generating functions have non-negative coefficients. Below we state and prove a generalization. Remarkably, Theorems [3 and [7 do not seem to have been previously noted.

Notation. We will denote the coefficient of $x_{1}^{m_{1}} \cdots x_{k}^{m_{k}}$ in $R_{n}\left(c ; x_{1}, \ldots, x_{k}\right)$ by

$$
\left\langle\begin{array}{c}
n \\
m_{1} \cdots m_{k}
\end{array}\right\rangle_{c}
$$

and the coefficient of the same monomial in $S_{n}\left(c ; x_{1}, \ldots, x_{k}\right)$ by

$$
\left[\begin{array}{c}
n \\
m_{1} \cdots m_{k}
\end{array}\right]_{c}
$$

\subsection{The univariate case.}

Theorem 3. Let $c>1$. Then all the coefficients of $R_{n}(c ; x)$ are non-negative. Indeed the coefficients of $x^{n}, x^{n-2}, \ldots, x^{-n+2}, x^{-n}$ are positive, while the other coefficients are zero. The same is true of $S_{n}$ in place of $R_{n}$.

Proof. By definition, $\left[\begin{array}{l}n \\ k\end{array}\right]_{c}$ is the coefficient of $x^{k}$ in $U_{n}((c / 2)(x+1 / x))$. The recurrence (10) gives the following recurrence for $\left[\begin{array}{l}n \\ k\end{array}\right]_{c}$ :

$$
\left.\left[\begin{array}{c}
n+1 \\
k
\end{array}\right]_{c}=c\left(\left[\begin{array}{c}
n \\
k-1
\end{array}\right]_{c}+\left[\begin{array}{c}
n \\
k+1
\end{array}\right]_{c}\right]\right)-\left[\begin{array}{c}
n-1 \\
k
\end{array}\right]_{c} .
$$

Now we shall show that the following always hold:

(a):

(b):

$$
\left[\begin{array}{l}
n \\
k
\end{array}\right]_{c} \geq 0
$$

(c):

$$
\left[\begin{array}{l}
n \\
k
\end{array}\right]_{s} \geq \max \left(\left[\begin{array}{l}
n-1 \\
k-1
\end{array}\right]_{c},\left[\begin{array}{l}
n-1 \\
k+1
\end{array}\right]_{c}\right)
$$

$$
\left[\begin{array}{l}
n \\
k
\end{array}\right]_{c} \geq\left[\begin{array}{c}
n-2 \\
k
\end{array}\right]
$$


with strict inequality in (a), (b), and (c) if and only if $n-k$ is even. The proof proceeds by induction; first the induction step (we assume throughout that $n-k$ is even; all the quantities involved are obviously 0 otherwise), we also drop the subscript $c$ for typographical reasons.

By induction (property (b)),

$$
\left[\begin{array}{c}
n \\
k+1
\end{array}\right]>\left[\begin{array}{c}
n-1 \\
k
\end{array}\right]
$$

Also,

It follows that

$$
\left[\begin{array}{c}
n \\
k-1
\end{array}\right]>\left[\begin{array}{c}
n-1 \\
k
\end{array}\right]
$$

$$
\left[\begin{array}{c}
n-1 \\
k
\end{array}\right]<\min \left(\left[\begin{array}{c}
n \\
k+1
\end{array}\right],\left[\begin{array}{c}
n \\
k-1
\end{array}\right]\right) \text {. }
$$

Hence, by the recurrence (12) it follows that

$$
\left[\begin{array}{c}
n+1 \\
k
\end{array}\right]>\max \left(\left[\begin{array}{c}
n \\
k-1
\end{array}\right],\left[\begin{array}{c}
n \\
k+1
\end{array}\right]\right) .
$$

Properties (a) and (c) follow immediately.

For the base case of the induction we note that $\left[\begin{array}{l}0 \\ 0\end{array}\right]=1$, while $\left[\begin{array}{l}1 \\ 1\end{array}\right]=\left[\begin{array}{c}1 \\ -1\end{array}\right]=c>1$.

We have thus proved the result for $\left[\begin{array}{l}n \\ k\end{array}\right]$. The same proof does not work for $\left\langle\begin{array}{l}n \\ k\end{array}\right\rangle$, since the base case fails. Indeed, $\left\langle\begin{array}{l}0 \\ 0\end{array}\right\rangle=1$, while $\left\langle\begin{array}{l}1 \\ 1\end{array}\right\rangle=c / 2$, not necessarily greater than 1. Fortunately, the result for $\left\langle\begin{array}{l}n \\ k\end{array}\right\rangle$ follows from the result for $\left[\begin{array}{l}n \\ k\end{array}\right]$ together with the observation that

$$
T_{n}(x)=\frac{U_{n}(x)-U_{n-2}(x)}{2} .
$$

Eq. (13) implies that $\left\langle\begin{array}{l}n \\ k\end{array}\right\rangle=\left[\begin{array}{l}n \\ k\end{array}\right]-\left[\begin{array}{c}n-2 \\ k\end{array}\right]>0$, by (c) above.

To complete the picture, we note that:

\section{Theorem 4.}

$$
R_{n}(1 ; x)=\frac{1}{2}\left(x^{n}+\frac{1}{x^{n}}\right) .
$$

Proof. Let $x=\exp i \theta$. Then $\frac{1}{2}(x+1 / x)=\cos \theta$, and $R_{n}(1 ; x)=T_{n}\left(\frac{1}{2}(x+1 / x)\right)=$ $\cos n \theta=\frac{1}{2}\left(x^{n}+1 / x^{n}\right)$.

Remark 5. For $c<-1$ it is true that all the coefficients of $R_{n}(c ;$.$) and S_{n}(c ;$.$) have$ the same sign, but the sign is $(-1)^{n}$. For $|c|<1$, the result is completely false. For $c$ imaginary, the result is true. I am not sure what happens for general complex $c$.

Bochner's Theorem states that a function $\omega$ on $\mathbb{R}$ is the Fourier transform of a non-negative function if and only if it is positive semi-definite; that is, for any real numbers $t_{1}, \ldots, t_{k}$ and complex numbers $z_{1}, \ldots, z_{k}$ the following inequality holds:

$$
\sum_{i, j \leq k} \omega\left(x_{i}-x_{j}\right) z_{i} \overline{z_{j}} \geq 0
$$

Since, as observed below (Section 3), the Fourier transform of $R_{n}(c ; x)$ is $T_{n}(c \cos \theta)$ and that of $S_{n}(c ; x)$ is $U_{n}(c \cos x)$ we have the following

Corollary 6. The functions $T_{n}(c \cos \theta)$ and $U_{n}(c \cos \theta)$ are positive semi-definite for $c \geq 1$. 
By the formula (11), we can write

$$
T_{n}\left(\frac{c}{2}\left(x+\frac{1}{x}\right)\right)=\frac{1}{2} \sum_{m=0}^{\left[\frac{n}{2}\right]}(-1)^{m} \frac{n}{n-m}\left(\begin{array}{c}
n-m \\
m
\end{array}\right) c^{n-2 m}\left(x+\frac{1}{x}\right)^{n-2 m} .
$$

Noting that

$$
\left(x+\frac{1}{x}\right)^{k}=\sum_{i=0}^{k}\left(\begin{array}{l}
k \\
i
\end{array}\right) x^{k-2 i}
$$

we obtain the expansion

$$
R_{n}(c ; x)=c^{n} \sum_{k=-n}^{n} x^{k} \sum_{m=0}^{\left[\frac{n}{2}\right]}\left(-\frac{1}{c^{2}}\right)^{m} \frac{n}{n-m}\left(\begin{array}{c}
n-m \\
m
\end{array}\right)\left(\begin{array}{c}
n-2 m \\
(n-2 m-k) / 2
\end{array}\right),
$$

where it is understood that $\left(\begin{array}{l}a \\ b\end{array}\right)$ is 0 if $b<0$, or $b>a$, or $b \notin \mathbf{Z}$. A similar formula for $S_{n}$ can be obtained by using Eq. (13).

2.2. The multivariate case. The proof of Theorem 3 goes through without too much change to prove Theorem 7 below. It is, however, quite apparent (from Theorem 1 as well as experiment) that the hypothesis $c \geq k$ is far from sharp. Experiment shows that it is not sufficient to assume that $c \geq 1$, but I conjecture that it is enough to assume that $c=O\left(k^{\epsilon}\right)$ for any $\epsilon$.

Theorem 7. Let $c \geq k$. Then all the coefficients of $R_{n}\left(c ; x_{1}, \ldots, x_{k}\right)$ are nonnegative. The same is true of $S_{n}$ in place of $R_{n}$.

Proof. The proof is very similar to the proof of Theorem [3. So we will just state the main points. Firstly, the multivariate version of recurrence (12):

$$
\left[\begin{array}{c}
n+1 \\
m_{1} \cdots m_{k}
\end{array}\right]_{c}=\frac{c}{k} \sum_{i=1}^{k}\left[\begin{array}{c}
n \\
m_{1} \cdots m_{i} \pm 1 \cdots m_{k}
\end{array}\right]_{c}-\left[\begin{array}{c}
n-1 \\
m_{1} \cdots m_{k}
\end{array}\right]_{c} .
$$

Secondly, the analogue of condition (b) from the proof of Theorem 3

$$
\left[\begin{array}{c}
n+1 \\
m_{1} \cdots m_{k}
\end{array}\right]_{c} \geq \max _{i=1}^{k}\left[\begin{array}{c}
n \\
m_{1} \cdots m_{i} \pm 1 \cdots m_{k}
\end{array}\right]_{c} .
$$

\section{Limiting Distribution OF COEFFICIENTS}

While the formula (16) is completely explicit, and a similar (though more cumbersome) expression could be obtained for $R_{n}\left(c ; x_{1}, \ldots, x_{k}\right)$, for many purposes it is more useful to have a limiting distribution formula as given by Theorem 8 below. To set up the framework, we note that since all the coefficients of $R_{n}\left(c ; x_{1}, \ldots, x_{k}\right)$ are non-negative (according to Theorem 17), they can be thought of as defining a probability distribution on the integer lattice $\mathbf{Z}^{k}$, defined by

$$
p\left(l_{1}, \ldots, l_{k}\right)=\left[x_{1}^{l_{1}} x_{2}^{l_{2}} \cdots x_{k}^{l_{k}}\right] R\left(c ; x_{1}, \ldots, x_{k}\right) / R(c ; 1, \ldots, 1)
$$

(where the square brackets mean that we are extracting the coefficients of the bracketed monomial). Call the resulting probability distribution $\mathcal{P}_{n}(c ; \mathbf{z})$, where $\mathbf{z}$ now denotes a $k$-dimensional vector. 
Theorem 8. With notation as above, when $c>1$, the probability distributions $\mathcal{P}_{n}(c ; \mathbf{z} / \sqrt{n})$ converge to a normal distribution on $\mathbf{R}^{k}$, whose mean is $\mathbf{0}$, and whose covariance matrix $C$ is diagonal, with entries

$$
\sigma^{2}=\frac{c}{k}\left[1+\left(\frac{c+1}{c-1}\right)^{1 / 2}\right] .
$$

To prove Theorem 8 we will use the method of characteristic functions (Fourier transforms), and more specifically at first the Continuity Theorem ([FellerII, Chapter XV.3, Theorem 2]):

Theorem 9. In order that a sequence $\left\{F_{n}\right\}$ of probability distributions converges properly to a probability distribution $F$, it is necessary and sufficient that the sequence $\left\{\phi_{n}\right\}$ of their characteristic functions converges pointwise to a limit $\phi$, and that $\phi$ is continuous in some neighborhood of the origin.

In this case $\phi$ is the characteristic function of $F$. (Hence $\phi$ is continuous everywhere and the convergence $\phi_{n} \rightarrow \phi$ is uniform on compact sets.)

The characteristic function $\phi_{n}$ of $\mathcal{P}_{n}(c ; \mathbf{z})$ is simply

$$
R_{n}\left(c ; \exp \left(i \theta_{1}\right), \ldots, \exp \left(i \theta_{k}\right)\right) / R(c ; 1, \ldots, 1) .
$$

By definition of $R_{n}$,

$$
\begin{aligned}
R_{n}\left(c ; \exp \left(i \theta_{1}\right), \ldots, \exp \left(i \theta_{k}\right)\right) & =T_{n}\left(\frac{c}{k} \sum_{j=1}^{k} \cos \theta_{j}\right) \\
\left.R_{n}(c ; 1, \ldots, 1)\right) & =T_{n}\left(\frac{c}{k} \sum_{j=1}^{k} \cos 0\right)=T_{n}(c) .
\end{aligned}
$$

We now use the form of Eq. (77):

$$
T_{n}(x)=\frac{1}{2}\left(\left(x-\sqrt{x^{2}-1}\right)^{n}+\left(x+\sqrt{x^{2}-1}\right)^{n}\right) .
$$

Setting

$$
u=\sum_{j=1}^{k} \cos \frac{\theta_{j}}{\sqrt{n}}
$$

and

we get

$$
\boldsymbol{\theta}=\left(\theta_{1}, \ldots, \theta_{k}\right)
$$

Notice, however, that for $c>1$, the ratio of the second term in braces to the first is exponentially small as $n \rightarrow \infty$, since the first term grows like $\left(c+\sqrt{c^{2}-1}\right)^{n}$, while the second as $\left(c-\sqrt{c^{2}-1}\right)^{n}$ (since $\cos \frac{\theta_{j}}{\sqrt{n}} \rightarrow 1$ ). Since, for the same reason, $2 T_{n}(c)=\left(c+\sqrt{c^{2}-1}\right)^{n}[1+o(1)]$, we can write

$$
\phi_{n}\left(\frac{\boldsymbol{\theta}}{\sqrt{n}}\right)=\left[\frac{\frac{c}{k} u+\sqrt{\frac{c^{2}}{k^{2}} u^{2}-1}}{c+\sqrt{c^{2}-1}}\right]^{n}+o(1) .
$$


Substituting the Taylor expansions for the cosine terms (hidden in $u$ for typesetting reasons), we get

$$
u=k+\frac{1}{2 n}\langle\boldsymbol{\theta}, \boldsymbol{\theta}\rangle+o(1 / n)
$$

so

$$
\frac{c}{k} u=c+\frac{c}{2 k n}\langle\boldsymbol{\theta}, \boldsymbol{\theta}\rangle+o(1 / n) .
$$

A similar computation gives

$$
\frac{c^{2}}{k^{2}} u^{2}=c^{2}+\frac{c^{2}}{k n}\langle\boldsymbol{\theta}, \boldsymbol{\theta}\rangle+o(1 / n) .
$$

Substituting the last expansion into the square root, we see that

$$
\begin{aligned}
\sqrt{\frac{c^{2}}{k^{2}} u^{2}-1} & =\sqrt{c^{2}-1} \sqrt{1+\frac{1}{n}\left[\frac{c^{2}}{\left(c^{2}-1\right) k}\langle\boldsymbol{\theta}, \boldsymbol{\theta}\rangle+o\left(\frac{1}{n}\right)\right]} \\
& =\sqrt{c^{2}-1}\left[1+\frac{1}{2 n} \frac{c^{2}}{\left(c^{2}-1\right) k}\langle\boldsymbol{\theta}, \boldsymbol{\theta}\rangle\right]+o\left(\frac{1}{n}\right) .
\end{aligned}
$$

Adding Eq. (20) and collecting terms, we get

$$
\begin{aligned}
& \frac{c u+\sqrt{c^{2} u^{2}-k^{2}}}{k\left(c+\sqrt{c^{2}-1}\right)} \\
& \quad=1+\frac{1}{2 n k}\left(1+\frac{1}{c+\sqrt{c^{2}-1}}\right)\left(c+\frac{c^{2}}{\left(c^{2}-1\right)^{1 / 2}}\right)\langle\boldsymbol{\theta}, \boldsymbol{\theta}\rangle+o\left(\frac{1}{n}\right) .
\end{aligned}
$$

Performing some further simplifications, we see that

$$
\phi_{n}\left(\frac{\boldsymbol{\theta}}{\sqrt{n}}\right)=\exp \left(-\frac{1}{2} \boldsymbol{\theta}^{\perp} C \boldsymbol{\theta}\right)+o(1),
$$

where $C$ is the covariance matrix described in the statement of Theorem 8 , and Theorem 8 follows immediately.

Remark 10. As noted in [FellerII pp. 515-517], the proof of the central limit theorem as above is easily adapted to give a local limit theorem (on the convergence of densities). In the special case of Theorem 1 we easily recover the result of Sharp (Sharp01]).

Remark 11. It is also not difficult to show that the speed of convergence in Theorem 8 is linear in $n$.

\section{REFERENCES}

[FellerII] William Feller. An Introduction to Probability Theory and its Applications, Volume II, second edition, John Wiley, New York, 1971. MR 0270403 (42:5292)

[Rivin99] Igor Rivin. Growth in Free Groups (and other stories), arxiv.org preprint math.CO/9911076

[Rivlin90] Theodore J. Rivlin. Chebyshev Polynomials: From Approximation Theory to Algebra and Number Theory, second edition, Wiley Interscience, 1990. MR1060735 (92a:41016)

[Sharp01] Richard Sharp. Local limit theorems for free groups, Math. Ann. 321(4), 2001, pp. 889904. MR 1872533 (2002k:20039)

[Schur73] Issai Schur. Arithmetischen über die Tschebyscheffschen Polynome, in Gesammelte Abhandlungen, III, Springer, Berlin, 1973, pp. 422-453. MR0462891 (57:2858a)

Department of Mathematics, Temple University, Philadelphia, Pennsylvania 19122

E-mail address: rivin@math.temple.edu 